\title{
Uncovering the complexity of Tourette syndrome, little by little ${ }^{\dagger}$
}

Daniel A. Gorman and Elia Abi-Jaoude

\begin{abstract}
Summary
The aetiology of Tourette syndrome is highly complex and still poorly understood. In this issue, using data from a large, prospective, population-based cohort of children, Mathews et al examine associations of preand perinatal exposures with Tourette syndrome and other chronic tic disorders. Their work illustrates the
\end{abstract}

importance of environmental factors in the aetiology of neuropsychiatric conditions and the value of replication in science.

\section{Declaration of interest}

None.
Daniel A. Gorman (pictured) is an assistant professor in the Department of Psychiatry at the University of Toronto. He is a child psychiatrist whose work focuses on Tourette syndrome, attention-deficit hyperactivity disorder, child psychopharmacology and psychiatric education. Elia Abi-Jaoude is a research fellow in the Department of Psychiatry at the University of Toronto. His clinical and research interests include Tourette syndrome, self-regulation and critical thinking in medical education and clinical practice.

'Science, my boy, is made up of mistakes, but they are mistakes which it is useful to make, because they lead little by little to the truth.

Jules Verne, Journey to the Centre of the Earth

Tourette syndrome is considered a prototypical developmental neuropsychiatric disorder. ${ }^{1}$ Its defining symptoms, motor and vocal tics, are neurological but it is usually associated with one or more psychiatric conditions, especially attention-deficit hyperactivity disorder and obsessive-compulsive disorder. In addition, the tics often follow a well-described developmental course: onset in early childhood, increasing severity until approximately age 11 and for most patients a gradual improvement during adolescence. ${ }^{2}$ Despite these general patterns related to comorbidity and natural history, however, the variability of Tourette syndrome is remarkable. Patients differ enormously with respect to the nature, severity and course of their tics, the range of their comorbid conditions, the degree of their functional impairment and their response to treatment. It is hardly surprising, therefore, that the aetiology of Tourette syndrome is highly complex and still poorly understood, with genetic, epigenetic, neurobiological, environmental and psychosocial factors all contributing.

\section{The multistrike model of aetiology}

To account for the clinical complexity and heterogeneity that characterise Tourette syndrome, a 'multistrike model' of its aetiology has been proposed. ${ }^{3}$ In this model, Strike 1 represents a genetic vulnerability that results in structural and functional neurological changes. These neurological changes, in turn, predispose the individual to develop tics and related symptoms. Strike 2 represents disturbances in brain regions that support self-regulatory functions. When these brain regions function normally, they mount a compensatory response that serves to inhibit tic expression; consequently, a disturbance in their functioning compromises the compensatory response, resulting in increased tic severity. Strike 3 comprises a host of environmental

'See pp. 40-45, this issue factors that increase the risk of tics being expressed in genetically and neurologically vulnerable individuals. These factors include neuroendocrine, infectious, autoimmune, toxic and psychosocial influences. Moreover, they can be divided into (a) pre- and perinatal events that exert 'organisational' effects on brain networks that generate tics, and (b) postnatal events that exert 'activational' effects on these networks, thereby increasing the expression of tics. ${ }^{4}$

Although the multistrike model is a useful framework for guiding research, it is not particularly specific. In fact, it can probably be applied to all complex developmental neuropsychiatric disorders. ${ }^{3}$ Unfortunately, a more specific model of the aetiology of Tourette syndrome is not possible at this point, because at the level of each strike of the general model, research findings are disappointing or conflicting, require replication or have significant limitations. For example, even though twin and family studies have demonstrated that Tourette syndrome is highly heritable, no candidate genes have been replicated consistently, and recently the first genome-wide association study detected no genome-wide significant loci. ${ }^{5}$

Neuroimaging studies have yielded more promising results, suggesting that reduced caudate volume and thinning of motor and sensorimotor cortices reflect the neurological predisposition to tics. In addition, hypertrophy of cortical (dorsolateral prefrontal, orbitofrontal and parietal) and limbic (amygdala and hippocampus) regions and a smaller corpus callosum are thought to reflect compensatory neuroplastic changes. ${ }^{6}$ Almost all these studies have been cross-sectional, however, making it difficult to tease out underlying cause from compensatory response. Conflicting findings have also been reported, likely as a result of differences in sample size, demographic characteristics, tic severity, comorbid conditions, medication use, techniques for the acquisition and processing of images, and methods for data analysis. Nevertheless, the neuroimaging literature does suggest that tic formation results from a disordered striatum and related dysfunction in cortico-striato-thalamo-cortical circuits. This notion is further supported by neuropathological studies showing reduced number and altered distribution of inhibitory GABAergic neurons in the sensorimotor areas of the striatum. ${ }^{7}$

\section{Role of environmental risk factors}

Except for the controversy regarding the potential role of group A beta-haemolytic streptococcal (GABHS) infections in a subset of patients with Tourette syndrome or obsessive-compulsive disorder, ${ }^{4}$ less attention has been given to environmental factors 
that influence the expression of tics. The number of studies in this area is still relatively small, and limitations often include retrospective design, absence of a control group and use of clinical rather than population-based samples. Furthermore, although the findings have generated interesting hypotheses, most have not been replicated. Generation of hypotheses is of course critical for scientific research, but a literature made up of many unreplicated findings risks the adoption of premature conclusions or may be dismissed altogether. Researchers on both sides of the Atlantic have argued recently for greater emphasis on studies that aim to replicate previous findings, ${ }^{8,9}$ and it has been said that 'reproducibility . . . is at the core of "the spirit of science". ${ }^{10}$ In this issue of the Journal, the study by Mathews and colleagues ${ }^{11}$ exemplifies this spirit.

Using data from the Avon Longitudinal Study of Parents and Children (ALSPAC) - a prospective, prebirth cohort of all children born to close to 15000 women in Avon, UK - Mathews et al examine associations of pre- and perinatal exposures with Tourette syndrome and other chronic tic disorders. A primary objective of the study was to determine whether associations detected in previous studies could be replicated: maternal prenatal smoking and alcohol use, excessive vomiting during pregnancy, complications of delivery, low birth weight and young gestational age at birth. Perhaps surprisingly, an association with only one of these previously identified risk factors - alcohol use during pregnancy - was replicated. The authors also identified three potential risk factors that have not been previously reported: cannabis use during pregnancy, parity (being the first-born child) and inadequate weight gain during pregnancy. Based on the odds ratios and significance levels in both the Tourette syndrome and Tourette syndrome/chronic tic disorder groups, the most robust risk factor appears to be inadequate weight gain during pregnancy. All three new findings, however, now await replication.

As the authors themselves point out, the pattern of associations they identified does not suggest an obvious overall mechanism by which environmental risk factors exert their effects. For example, why should inadequate weight gain during pregnancy be such a strong risk factor for Tourette syndrome, but not low birth weight? Why should being the first-born child make a difference? It is tempting to apply Occam's razor in search of a unifying, parsimonious explanation for these findings, but doing so would probably be misguided. The aetiology of Tourette syndrome is a complex problem, and as H. L. Mencken said, 'For every complex problem there is an answer that is clear, simple, and wrong. Bearing in mind the multistrike model of Tourette syndrome and other complex developmental neuropsychiatric disorders, it is likely that environmental risk factors have their effects at different points in the cascade of genetic, epigenetic and neurobiological events that ultimately lead to the expression of tics and related symptoms.

Among the risk factors for Tourette syndrome/chronic tic disorder identified by Mathews et al, the one that is probably most easily explained is prenatal alcohol exposure, since it is associated with poor inhibitory control and has been shown to impair cortical development and plasticity. ${ }^{12}$ Thus, prenatal alcohol exposure could increase the risk of Tourette syndrome in predisposed individuals by disrupting the compensatory mechanisms that serve to inhibit tic expression. Caution is still warranted, however, regarding the role of prenatal alcohol exposure in the aetiology of Tourette syndrome, as epidemiological study findings have a high risk of confounding bias even with the best efforts to account for potential confounders. For instance, it could be that mothers with a genetic predisposition to Tourette syndrome are more likely to use alcohol during pregnancy, perhaps as a result of related problems with behavioural inhibition.
Despite the inherent risk of confounding bias and the relatively small sample size (50 participants with Tourette syndrome, 122 with Tourette syndrome/chronic tic disorder), the study by Mathews et al has many strengths that support the validity of its findings. These include the prospective, longitudinal design; ascertainment of participants from the general population; use of a conservative algorithm for data analysis; and careful procedures to control for potential confounders. Regarding this last issue, it is notable that controlling for socioeconomic status in univariate analyses tended to reduce the strength of associations of potential risk factors, rendering certain ones non-significant (maternal age and assisted delivery). This pattern highlights the contribution of social factors in the aetiology of Tourette syndrome, even though the disorder has a strong neurobiological basis and is even defined by neurological symptoms.

It is well recognised that stress and social context can influence tic expression in the moment, but evidence is also emerging regarding the influence of social factors on long-term tic severity. For example, psychosocial stress was found to modestly predict increased future tic severity in children and adolescents with chronic tics, ${ }^{13}$ and the effect was three times stronger in the context of a new GABHS infection. ${ }^{14}$ In addition, Mathews et al cite a previous (unpublished) finding that lower socioeconomic status was associated with an increased risk of developing Tourette syndrome/chronic tic disorder. These results are in keeping with evidence regarding the contribution of social factors in the aetiology of other neuropsychiatric conditions, such as schizophrenia, Alzheimer's disease and obsessive-compulsive disorder. ${ }^{15}$ Indeed, the important role of socioeconomic status ${ }^{16}$ and stress ${ }^{17}$ in disease has long been recognised.

\section{Conclusion}

The contemporary view of cutting-edge psychiatric research tends to prioritise new discoveries that reveal the secrets of the genome or the brain, whereas studies aiming to replicate previous findings or examine environmental factors are decidedly less glamorous. Rigorous replication studies, however, are an essential step in science, and environmental factors are no less important than biological ones with respect to the aetiology of mental illness as well as the development of effective therapies. Mathews et al should therefore be commended for their efforts to replicate previously reported associations of pre- and perinatal exposures with Tourette syndrome. By suggesting that a number of those associations may be mistaken, their results lead us a little closer to the truth.

\footnotetext{
Daniel A. Gorman, MD, FRCPC, Department of Psychiatry, University of Toronto and The Hospital for Sick Children, Toronto; Elia Abi-Jaoude, MSC, MD, FRCPC, Department of Psychiatry, University of Toronto and University Health Network (Toronto Western Hospital), Toronto, Canada
(Tepartment of Psychiatry, University of Toronto

Correspondence: Daniel A. Gorman, MD, FRCPC, The Hospital for Sick Children 555 University Avenue, Toronto M5G 1X8, Canada. Email: daniel.gorman@utoronto.ca

First received 16 Aug 2013, accepted 16 Sep 2013
}

\section{References}

1 Steinberg T, King R, Apter A. Tourette's syndrome: a review from a developmental perspective. ISr J Psychiatry Relat Sci 2010; 47: 105-9.

2 Leckman JF, Zhang H, Vitale A, Lahnin F, Lynch K, Bondi C, et al. Course of tic severity in Tourette's syndrome: the first two decades. Pediatrics 1998; 102: 14-9.

3 Spessot AL, Plessen KJ, Peterson BS. Neuroimaging of developmental psychopathologies: the importance of self-regulatory and neuroplastic processes in adolescence. Ann NY Acad Sci 2004; 1021: 86-104. 
4 Hoekstra PJ, Dietrich A, Edwards MJ, Elamin I, Martino D. Environmental factors in Tourette syndrome. Neurosci Biobehav Rev 2013; 37: 1040-9.

5 Scharf JM, Yu D, Mathews CA, Neale BM, Stewart SE, Fagerness JA, et al. Genome-wide association study of Tourette's syndrome. Mol Psychiatry 2013; 18: 721-8.

6 Plessen KJ, Bansal R, Peterson BS. Imaging evidence for anatomical disturbances and neuroplastic compensation in persons with Tourette syndrome. J Psychosom Res 2009; 67: 559-73.

7 Ganos C, Roessner V, Munchau A. The functional anatomy of Gilles de la Tourette syndrome. Neurosci Biobehav Rev 2013; 37: 1050-62.

8 Munafò MR, Flint J. How reliable are scientific studies? Br J Psychiatry 2010; 197: $257-8$.

9 Murad MH, Montori VM. Synthesizing evidence: shifting the focus from individual studies to the body of evidence. JAMA 2013; 309: 2217-8.

10 Santer BD, Wigley TM, Taylor KE. The reproducibility of observational estimates of surface and atmospheric temperature change. Science 2011; 334: 1232-3.

11 Mathews CA, Scharf JM, Miller LL, Macdonald-Wallis C, Lawlor DA, Ben-Shlomo $Y$. Association between pre- and perinatal exposures and Tourette syndrome or chronic tic disorder in the ALSPAC cohort. $\mathrm{Br} J$ Psychiatry 2014; 204: 40-5.
12 Lebel C, Mattson SN, Riley EP, Jones KL, Adnams CM, May PA, et al. A longitudinal study of the long-term consequences of drinking during pregnancy: heavy in utero alcohol exposure disrupts the normal processes of brain development. J Neurosci 2012; 32: 15243-51.

13 Lin $\mathrm{H}$, Katsovich L, Ghebremichael M, Findley DB, Grantz H, Lombroso PJ, et al. Psychosocial stress predicts future symptom severities in children and adolescents with Tourette syndrome and/or obsessive-compulsive disorder. J Child Psychol Psychiatry 2007; 48: 157-66.

14 Lin $\mathrm{H}$, Williams KA, Katsovich L, Findley DB, Grantz H, Lombroso PJ, et al. Streptococcal upper respiratory tract infections and psychosocial stress predict future tic and obsessive-compulsive symptom severity in children and adolescents with Tourette syndrome and obsessive-compulsive disorder. Biol Psychiatry 2010; 67: 684-91.

15 Eisenberg L. The social construction of the human brain. Am J Psychiatry 1995; 152: 1563-75.

16 Spencer N, Thanh TM, Louise S. Low income/socio-economic status in early childhood and physical health in later childhood/adolescence: a systematic review. Matern Child Health J 2013; 17: 424-31.

17 Cohen S, Janicki-Deverts D, Miller GE. Psychological stress and disease. JAMA 2007; 298: 1685-7.

Gilles de la Tourette syndrome was first described by Parisian doctor Georges Albert Édouard Brutus Gilles de la Tourette in 1885 Originally considered a rare medical curiosity, the syndrome of multiple tics is now regarded as a fairly common neurodevelopmental condition, affecting up to $1 \%$ of school-age children. Gilles de la Tourette described the clinical triad of tics, echolalia and coprolalia. The last is relatively rare and no longer features as a diagnostic criterion, despite the media obsession with obscene manifestations. French colleagues react with horror when they see their syndrome's name shortened to Tourette syndrome, even in 100-word texts. 